\title{
ESTIMATION OF FIRMS EFFICIENCIES USING A KALMAN FILTER AND STOCHASTIC EFFICIENCY MODEL
}

\author{
Tohru Ueda Kenichi Hoshino \\ Seikei University
}

(Received March 1, 2005; Revised July 28, 2005)

\begin{abstract}
The efficiencies of firms should be evaluated, based on their historical financial data. Here a process for evaluation is proposed. Each historical data set is reduced to a distribution function with a mean and a variance estimated by Kalman filter. Then, a stochastic efficiency model is applied to this reduced data. Then a new efficiency measure is proposed and compared with existing measures. An application is made to three kinds of firms.
\end{abstract}

Keywords: DEA, forecasting, Kalman filter

\section{Introduction}

The efficiency of firms should be evaluated from a variety of viewpoints, and thus Data Envelopment Analysis (DEA) is used as a method of evaluating firms with many kinds of inputs and outputs. Evaluation based on comparison among firms at a single time point reflects spatial variance, but firms have their history and have longitudinal data. Window analysis is well-known as an analytic method for longitudinal data. However, this method does not seem to be appropriate for analysis of future performance, but seems to be appropriate for analysis of past performance.

As usual DEA uses one value by each kind of inputs and outputs respectively, we must decide which value should be selected. In a stochastic efficiency model shown in [7] a variance of each kind of inputs and outputs should be estimated.

The Kalman filter gives the minimum mean squared error of forecasts, that is, this is appropriate for analysis of future performance and gives not only an estimate of future value, but also an estimate of its variance. We can use these values as parameters of a stochastic efficiency model. We propose a new measure of the efficiency score called the modified efficiency score and compare it with existing measures.

\section{The Kalman Filter}

The Kalman filter is presented by system equations and an observation equation as shown in [5]. In this paper system equations at time $t$ are given by

$$
\mathbf{x}(t+1)=\dot{F} \mathbf{x}(t)+G U(t)
$$

and an observation equation is given by

$$
y(t)=H(t)^{t} \mathbf{x}(t)+w(t)
$$


where the superscript, $t$, is not time $t$, but means transposition, and

$$
\begin{aligned}
& \mathbf{x}(t)^{t}=(T(t), T(t-1), L(t)), \\
& T(t) \text { : an element presenting trend at time } t \text {, } \\
& \nabla^{k} T(t)=u(t) \sim N\left(0, \tau^{2}\right) ; \nabla T(t)=T(t)-T(t-1), \\
& \text { ( } k=2 \text { is used in this paper.) } \\
& F=\left[\begin{array}{ccc}
2 & -1 & 0 \\
1 & 0 & 0 \\
0 & 0 & 1
\end{array}\right] \text {, } \\
& G^{t}=\left[\begin{array}{lll}
1 & 0 & 0 \\
0 & 0 & 0
\end{array}\right], U(t)^{t}=(u(t), 0), \\
& H(t)^{t}=(1,0, d(t, N)) ; w(t) \sim N\left(0, \omega^{2}\right), \\
& d(t, N)= \begin{cases}1 & : t=N, \\
0 & : t \neq N .\end{cases}
\end{aligned}
$$$$
L(t) \text { : an element presenting effect of an accidental event at time } t \text {, }
$$

The expectation and variance of forecasts are given by

$$
\begin{aligned}
& y(t \mid t-1)=H(t)^{t} \mathbf{x}(t \mid t-1) \\
& R(t \mid t-1)=H(t)^{t} P(t \mid t-1) H(t)+\omega^{2}
\end{aligned}
$$

where $A(t \mid t-1)$ is a forecast of $A(t)$ at time $(t-1)$, for example, $y(t \mid t-1)$ and $R(t \mid t-1)$ are the expectation and variance of forecasts of $y(t)$ at time $(t-1)$ and these values are renewed as follows and used in a stochastic efficiency model:

\section{(Renewal process on time)}

$$
\begin{aligned}
& \mathbf{x}(t \mid t-1)=F \mathbf{x}(t-1 \mid t-1) \\
& P(t \mid t-1)=F P(t-1 \mid t-1) F^{t}+G Q G^{t}
\end{aligned}
$$

where $Q$ is a variance-covariance matrix of $U$.

\section{(Modification based on observed values)}

$$
\begin{aligned}
& \mathbf{x}(t \mid t)=\mathbf{x}(t \mid t-1)+K(t)\{y(t)-y(t \mid t-1)\} \\
& P(t \mid t)=P(t \mid t-1)-K(t) H(t)^{t} P(t \mid t-1) \\
& K(t)=P(t \mid t-1) H(t) /\left\{H(t)^{t} P(t \mid t-1) H(t)+\omega^{2}\right\}
\end{aligned}
$$

Here we suppose that an accidental event occurred at time $N$, but some accidental event may occur.

We insist that such Kalman filter as shown in (1)-(12) should be used for evaluation of future performance.

\section{Stochastic Efficiency Model}

In usual DEA each Decision Making Unit (DMU) $O(=1,2, \ldots, n)$ has deterministic inputs $\mathbf{X}_{o}=\left(x_{1 o}, x_{2 o}, \ldots, x_{m o}\right)$ and deterministic outputs $\mathbf{Y}_{o}=\left(y_{1 o}, y_{2 o}, \ldots, y_{s o}\right)$ and its efficiency in Charnes, Cooper and Rhodes [4] (CCR) model is measured by

$$
\max \sum_{r} u_{r} y_{r o} / \sum_{i} v_{i} x_{i o}
$$


where

$$
\sum_{r} u_{r} y_{r j} / \sum_{i} v_{i} x_{i j} \leq 1 \quad(j=1,2, \ldots, n)
$$

However, in this paper the case where inputs and outputs are not deterministic is discussed. Assume that for each DMU $O(=1,2, \ldots, n)$ its inputs have multivariate normal distributions with mean, $\mathbf{X}_{o}=\left(x_{1 o}, x_{2 o}, \ldots, x_{m o}\right)^{t}$, and variations, $\Delta \mathbf{X}_{o}=\left(\Delta x_{1 o}, \Delta x_{2 o}, \ldots, \Delta x_{m o}\right)^{t}$ and its outputs have multivariate normal distributions with mean, $\mathbf{Y}_{o}=\left(y_{1 o}, y_{2 o}, \ldots, y_{s o}\right)^{t}$, and variations, $\Delta \mathbf{Y}_{o}=\left(\Delta y_{1 o}, \Delta y_{2 o}, \ldots, \Delta y_{s o}\right)^{t}$, where their variance-covariance matrix is given by $\Sigma_{o}$ and the confidence region of stochastic variations, $\delta \equiv\left(\Delta \mathrm{X}_{o}, \Delta \mathrm{Y}_{o}\right)$, at probability level $\alpha$ is given by

$$
S_{\alpha}=\left\{\delta \mid \delta \Sigma_{o}^{-1} \delta^{t} \leq \chi_{m+s}^{2}(\alpha)\right\}
$$

and let the worst inputs and outputs at probability level $\alpha$ ge

$$
\left(\mathbf{X}_{o \alpha}, \mathbf{Y}_{o \alpha}\right)=\left(\mathbf{X}_{o}+\Delta \mathbf{X}_{o}, \mathbf{Y}_{o}-\Delta \mathbf{Y}_{o} \mid \delta \Sigma_{o}^{-1} \delta^{t} \leq \chi_{m+s}^{2}(\alpha)\right)
$$

where $\chi_{m+s}^{2}(\alpha)$ is an $\alpha$ percentile of the $\chi^{2}$ distribution . Let the minimum efficiency score obtained within $S_{\alpha}$ be $W_{\alpha}$.

When a number of DMUs, $n$, is small, compared with a sum, $(m+s)$, of numbers of inputs and outputs, relatively many DMUs may be evaluated as efficient in the usual DEA. On the lines of [7], we calculate the maximum $\alpha_{\max }$ which gives $W_{\alpha}=1$ within $S_{\alpha}$. The more $\alpha_{\max }$ is, the more robust the efficient state is. For efficient DMU in CCR model this problem can be formulated as follows:

\section{[Problem 1]}

$$
\begin{array}{ll}
\max & \alpha \\
\text { subject to } & W_{\boldsymbol{\alpha}}=\mathbf{u}^{t} \mathbf{Y}_{o \alpha}=1 \\
& \mathbf{v}^{t} \mathbf{X}_{o \alpha}=1 \\
& \mathbf{v}^{t} \mathbf{X}_{j} \geq \mathbf{u}^{t} \mathbf{Y}_{j} ; j \neq o \\
& \delta \Sigma_{o}^{-1} \delta^{t} \leq \chi_{m+s}^{2}(\alpha)
\end{array}
$$

where $\mathbf{X}_{j}$ and $\mathbf{Y}_{j}$ are inputs and outputs of DMU $j$.

\section{New Efficiency Measure}

Solutions of Problem 1 give the efficiency score, $W_{\alpha}=1$. Therefore in order to distinguish many DMU with the efficiency score, 1 , we propose a new efficiency measure, $\tilde{W}_{o}$, using multipliers $\mathbf{v}^{*}$ and $\mathbf{u}^{*}$ obtained by solving Problem 1 :

$$
\tilde{W}_{o}=\left(\mathbf{u}^{* t} \mathbf{Y}_{o}\right) /\left(\mathbf{v}^{* t} \mathbf{X}_{o}\right)
$$

If $W_{\alpha}=1, \mathbf{v}^{* t} \mathbf{X}_{o \alpha}=\mathbf{v}^{* t}\left(\mathbf{X}_{o}+\Delta \mathbf{X}_{o}\right)=1$ and $\mathbf{u}^{* t} \mathbf{Y}_{o \alpha}=\mathbf{u}^{* t}\left(\mathbf{Y}_{o}-\Delta \mathbf{Y}_{o}\right)=1$. Then $\mathbf{v}^{* t} \mathbf{X}_{o} \leq 1$ and $\mathbf{u}^{* t} \mathbf{Y}_{o} \geq 1$. Therefore, this measure, $\tilde{W}_{o}$, gives a larger efficiency score than 1 for semi-positive $\delta$ (all are not zero), where for inefficient DMU the same efficiency scores as CCR model are given.

\section{Application}

The following three data groups were studied.

(1) 31 electric device manufacturers

(2) 19 department stores 
(3) 38 supermarkets

We use two kinds of indices as the management indices. The first indices were used in [8] as an altered discriminant function of $\mathrm{Z}$ value which was proposed by $\mathrm{E}$. I. Altman [1]. The alternative indices were selected for altering of the first indices.

\subsection{First indices}

The following indices are used for evaluation.

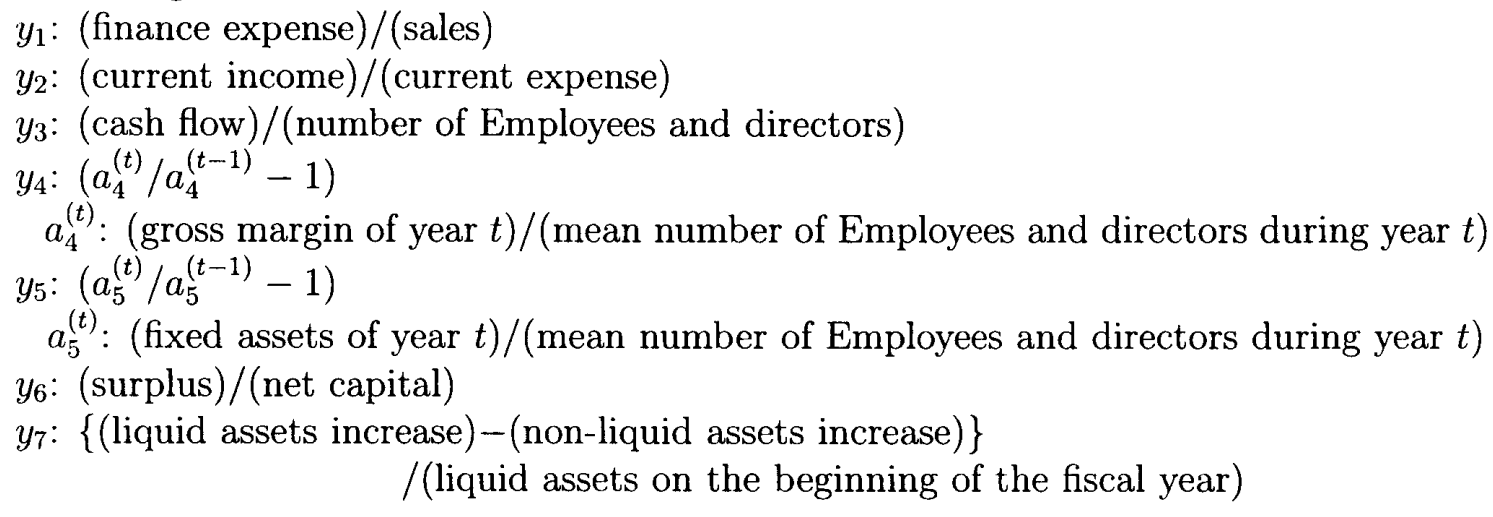

In DEA these indices are treated as outputs, where all inputs are assumed to be equal to one and $y_{j}(j=1,7)$ are replaced by ([maximum of them over all DMUs]- $\left[y_{j}\right.$ of the targeted DMU]).

We forecasted each index value of 2001, using indices values from 1986 to 2000 based on the Kalman filter represented by (1)-(12).

Coefficients of variation, that is, $C V=\sqrt{R(2001 \mid 2000)} / y(2001 \mid 2000)$ are shown in Table 1 and 2, where $R(n \mid n-1)$ and $y(n \mid n-1)$ are given by (9) and (10). Figure 1 shows variation of index $y_{1}$ at Mabuchi-Motor, where $R(2001 \mid 2000)=3.06, y(2001 \mid 2000)=2.39$ and $C V=0.732$ for $y_{1}$. As shown in these tables and figure, many forecasts have large variations, because corresponding observed values have large variations. However, we must be ready for such large variation and we use $R(2001 \mid 2000)$ and $y(2001 \mid 2000)$ for estimation of $\alpha_{\max }$.

Evaluation results for CCR efficient DMUs of each data groups are shown in Table 3, 4 and 5, where super-efficiency scores proposed by [2] are also shown in these tables for the purpose of comparison.

We think that $\alpha_{\max }$ is not appropriate as a measure for efficiency comparison, because several DMUs have a value zero. Super-efficiency scores can take such very large values as Toshibatec in Table 3 . However, our measure, $\tilde{W}_{o}$, take more moderate values than superefficiency scores. This may be an advantage of our measure. However, though Sogo and Mycal went down, they were evaluated as efficient DMUs. Therefore the alternative indices are used in the next session. 
Table 1: Forecasted coefficient of variation of each index for data group (1)

\begin{tabular}{|c|c|c|c|c|c|c|c|}
\hline & $\mathrm{y}_{1}$ & $\mathrm{y}_{2}$ & $\mathrm{y}_{3}$ & $\mathrm{y}_{4}$ & $\mathrm{y}_{5}$ & $\mathrm{y}_{6}$ & $\mathrm{y}_{7}$ \\
\hline msc.panasonic & 0.467 & 0.049 & 0.014 & 0.432 & 0.875 & 0.021 & 1.691 \\
\hline Toshibatec & 0.108 & 0.049 & 0.012 & 0.292 & 0.080 & 0.047 & 0.218 \\
\hline Mabuchi-Motor & 0.732 & 0.046 & 0.005 & 0.777 & 0.223 & 0.021 & 0.257 \\
\hline Matsushita & 0.222 & 0.048 & 0.015 & 0.181 & 0.312 & 0.016 & 0.142 \\
\hline Sharp & 0.216 & 0.046 & 0.008 & 0.573 & 0.502 & 0.030 & 0.853 \\
\hline Sony & 0.334 & 0.051 & 0.012 & 0.308 & 0.275 & 0.028 & 8.477 \\
\hline Aiwa & 1.665 & 0.056 & 0.010 & 0.188 & 0.664 & 0.046 & 0.246 \\
\hline Sanyo & 0.192 & 0.047 & 0.012 & 0.178 & 1.208 & 0.029 & 0.251 \\
\hline kme.panasonic & 0.192 & 0.048 & 0.015 & 5.951 & 0.396 & 0.026 & 1.225 \\
\hline Pioneer & 0.466 & 0.048 & 0.022 & 0.253 & 0.264 & 0.107 & 1.455 \\
\hline Columbia & 0.462 & 0.058 & 0.039 & 0.665 & 0.907 & 0.066 & 0.150 \\
\hline JvcVictor & 0.438 & 0.049 & 0.017 & 0.828 & 0.568 & 0.025 & 0.200 \\
\hline Foster & 0.363 & 0.050 & 0.012 & 0.173 & 0.319 & 0.035 & 0.876 \\
\hline Clarion & 0.120 & 0.053 & 0.008 & 0.352 & 0.471 & 0.049 & 0.243 \\
\hline Marantz & 0.094 & 0.051 & 0.012 & 0.344 & 0.271 & 0.027 & 0.150 \\
\hline Yokowo & 0.136 & 0.050 & 0.022 & 19.03 & 0.988 & 0.035 & 0.310 \\
\hline Zojirushi & 0.248 & 0.055 & 0.019 & 0.222 & 1.951 & 0.021 & 0.285 \\
\hline Teak & 0.153 & 0.055 & 0.008 & 0.220 & 0.323 & 0.041 & 0.391 \\
\hline mke.panasonic & 0.218 & 0.048 & 0.007 & 0.456 & 5.999 & 0.032 & 0.177 \\
\hline TOA & 0.104 & 0.058 & 0.017 & 0.159 & 0.185 & 0.036 & 0.990 \\
\hline Nakamichi & 0.542 & 0.053 & 0.009 & 0.147 & 0.636 & 0.085 & 0.511 \\
\hline mrc.panasonic & 0.179 & 0.047 & 0.037 & 0.106 & 3.194 & 0.022 & 0.163 \\
\hline Rion & 0.131 & 0.056 & 0.016 & 35.16 & 0.132 & 0.024 & 0.153 \\
\hline Enplas & 0.200 & 0.045 & 0.007 & 0.231 & 0.256 & 0.029 & 0.26 \\
\hline Hitachi & 2.243 & 0.047 & 0.017 & 0.245 & 0.513 & 0.023 & 0.295 \\
\hline Toshiba & 1.944 & 0.046 & 0.011 & 1.410 & 0.326 & 0.030 & 0.142 \\
\hline Melco & 1.491 & 0.045 & 0.068 & 7.726 & 0.467 & 0.031 & 0.147 \\
\hline Fujielectric & 0.614 & 0.054 & 0.012 & 0.086 & 0.195 & 0.047 & 1.285 \\
\hline NEC & 0.883 & 0.051 & 0.011 & 0.123 & 0.724 & 0.038 & 0.492 \\
\hline Fujitsu* & - & 0.050 & 0.059 & 0.250 & 0.235 & 0.031 & 1.422 \\
\hline Oki & 0.799 & 0.054 & 0.010 & 0.078 & 0.517 & 0.087 & 0.203 \\
\hline mean of CV & 0.532 & 0.05 & 0.018 & 2.489 & 0.773 & 0.038 & 0.763 \\
\hline Because $E(y)$ & $0, C V$ & & & & \\
\hline
\end{tabular}

*Because $E\left(y_{1}\right)=0, C V\left(y_{1}\right)$ cannot be calculated.

Table 2: Mean forecasted coefficient of variation of each index by each group

\begin{tabular}{|l|c|c|c|c|c|c|c|}
\hline & $\mathrm{y}_{1}$ & $\mathrm{y}_{2}$ & $\mathrm{y}_{3}$ & $\mathrm{y}_{4}$ & $\mathrm{y}_{5}$ & $\mathrm{y}_{6}$ & $\mathrm{y}_{7}$ \\
\hline group (1) & 0.532 & 0.050 & 0.018 & 2.489 & 0.773 & 0.038 & 0.763 \\
\hline group (2) & 0.560 & 0.049 & 0.039 & 1.430 & 2.357 & 0.051 & 0.967 \\
\hline group (3) & 0.515 & 0.056 & 0.054 & 3.273 & 1.466 & 0.066 & 0.889 \\
\hline
\end{tabular}




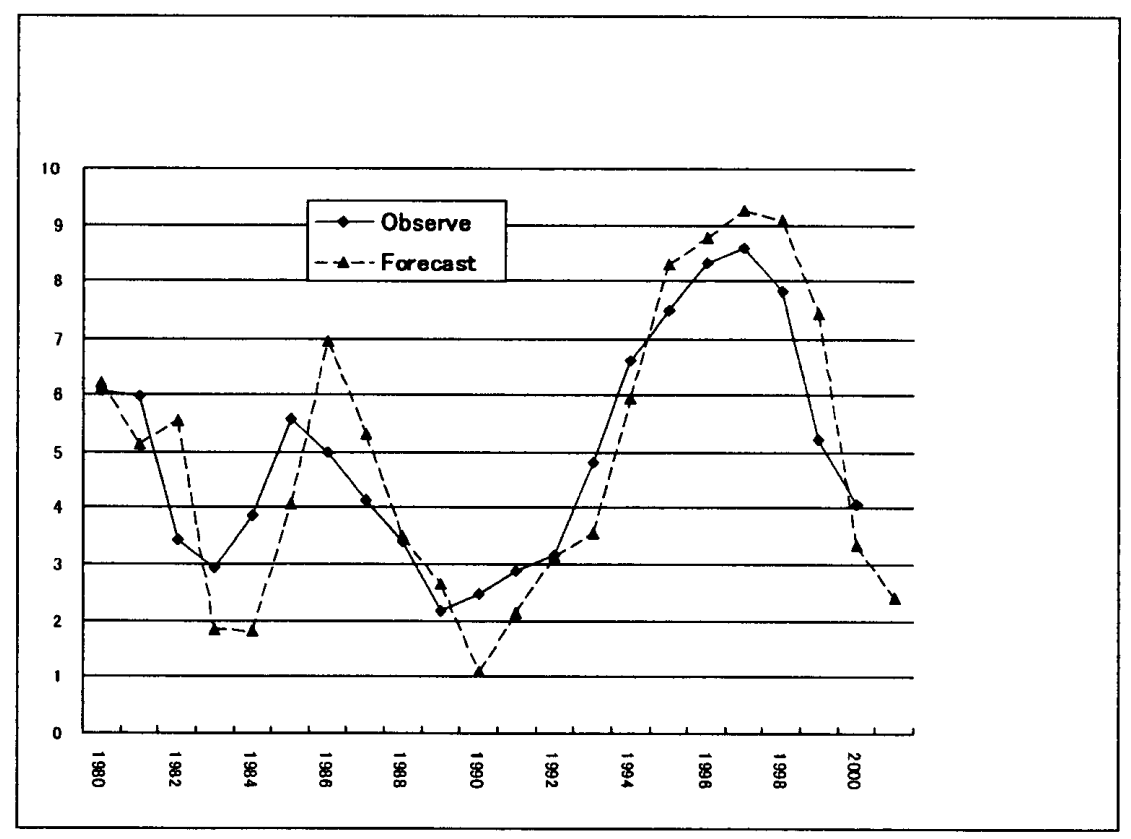

Figure 1: Variation of $y_{1}$ at Mabuchi-Motor

Table 3: Comparison among efficiency measures for data group (1)

\begin{tabular}{|c|c|c|c|c|c|c|}
\hline Rank & \multicolumn{2}{|c|}{$\alpha_{\max }$} & \multicolumn{2}{c|}{ Super-efficiency } & \multicolumn{2}{c|}{ Proposed measure } \\
\hline 1 & Toshibatec & $84.82 \%$ & Toshibatec & 3.846 & Mabuchi-motor & 1.860 \\
\hline 2 & Mabuchi-motor & $51.55 \%$ & Mabuchi-motor & 2.546 & Nakamichi & 1.813 \\
\hline 3 & Matsushita & $9.54 \%$ & Nakamichi & 2.116 & Toshibatec & 1.758 \\
\hline 4 & Nakamichi & $3.83 \%$ & Matsushita & 1.550 & Matsushita & 1.694 \\
\hline 5 & Toshiba & $1.30 \%$ & Toshiba & 1.454 & Toshiba & 1.586 \\
\hline 6 & Marantz & $0.00 \%$ & Marantz & 1.074 & Marantz & 1.076 \\
\hline 7 & Fujitsu & $0.00 \%$ & Melco & 1.050 & Melco & 1.056 \\
\hline 8 & Hitachi & $0.00 \%$ & Fujitsu & 1.032 & Fujitsu & 1.033 \\
\hline 9 & Melco & $0.00 \%$ & Hitachi & 1.024 & Hitachi & 1.025 \\
\hline 10 & Msc.Panasonic & $0.00 \%$ & Msc.panasonic & 1.021 & Msc.panasonic & 1.020 \\
\hline 11 & Enplas & $0.00 \%$ & Enplas & 1.008 & Enplas & 1.008 \\
\hline
\end{tabular}


Table 4: Comparison among efficiency measures for data group (2)

\begin{tabular}{|c|c|c|c|c|c|c|}
\hline Rank & \multicolumn{2}{|c|}{$\alpha_{\max }$} & \multicolumn{2}{c|}{ Super-efficiency } & Proposed measure \\
\hline 1 & Izutsuya & $0.80 \%$ & Izutsuya & 1.906 & Izutsuya & 1.535 \\
\hline 2 & Hankyu-Dept & $0.39 \%$ & Hankyu-Dept & 1.395 & Hankyu-Dept & 1.452 \\
\hline 3 & Matsuzakaya & $0.12 \%$ & Isetan & 1.331 & Matsuya & 1.336 \\
\hline 4 & Matsuya & $0.03 \%$ & Matsuya & 1.318 & Isetan & 1.306 \\
\hline 5 & Sogo & $0.01 \%$ & Daimaru & 1.233 & Nagano-Tokyu & 1.292 \\
\hline 6 & Isetan & $0.01 \%$ & Nagano-Tokyu & 1.212 & Daimaru & 1.267 \\
\hline 7 & Nagano-Tokyu & $0.00 \%$ & Matsuzakaya & 1.198 & Matsuzakaya & 1.236 \\
\hline 8 & Daimaru & $0.00 \%$ & Platz-Kintetsu & 1.154 & Sogo & 1.153 \\
\hline 9 & Platz-Kintetsu & $0.00 \%$ & Sogo & 1.141 & Iwataya & 1.147 \\
\hline 10 & Iwataya & $0.00 \%$ & Iwataya & 1.099 & Platz-Kintetsu & 1.145 \\
\hline 11 & Tokyu-Depart & $0.00 \%$ & Tokyu-Depart & 1.078 & Tokyu-Depart & 1.078 \\
\hline 12 & Daiwa-Dp & $0.00 \%$ & Daiwa-Dp & 1.044 & Takashimaya & 1.052 \\
\hline 13 & Takashimaya & $0.00 \%$ & Takashimaya & 1.034 & Daiwa-Dp & 1.043 \\
\hline 14 & Saikaya & $0.00 \%$ & Saikaya & 1.029 & Saikaya & 1.034 \\
\hline
\end{tabular}

Table 5: Comparison among efficiency measures for data group (3)

\begin{tabular}{|c|c|c|c|c|c|c|}
\hline Rank & \multicolumn{2}{|c|}{$\alpha_{\max }$} & \multicolumn{2}{c|}{ Super-efficiency } & \multicolumn{2}{c|}{ Proposed measure } \\
\hline 1 & Itoyokado & $11.17 \%$ & Itoyokado & 2.070 & Yamazawa & 1.873 \\
\hline 2 & Yorkbeni & $0.67 \%$ & Yamazawa & 1.822 & Jujiya & 1.837 \\
\hline 3 & Jujiya & $0.19 \%$ & Jujiya & 1.817 & Itoyokado & 1.547 \\
\hline 4 & Seiyu & $0.01 \%$ & Yorkbeni & 1.509 & Yorkbeni & 1.529 \\
\hline 5 & Marukyo & $0.00 \%$ & Seiyu & 1.242 & Seiyu & 1.228 \\
\hline 6 & Yamazawa & $0.00 \%$ & Marukyo & 1.222 & Marukyo & 1.205 \\
\hline 7 & SuperDaiei & $0.00 \%$ & SuperDaiei & 1.191 & SuperDaiei & 1.181 \\
\hline 8 & Taiyo & $0.00 \%$ & Taiyo & 1.181 & U-Store & 1.179 \\
\hline 9 & U-Store & $0.00 \%$ & U-Store & 1.152 & Taiyo & 1.165 \\
\hline 10 & Mycal & $0.00 \%$ & Mycal & 1.123 & Mycal & 1.127 \\
\hline
\end{tabular}




\subsection{The alternative indices}

The following indices are used for evaluation on the line of [6].

$z_{1}=\mathrm{ROA}:($ return $) /$ (assets)

$z_{2}$ : (operating profits)/(sales)

$z_{3}:$ (sales) $/$ (assets)

$z_{4}$ : (current assets)/(current liability)

$z_{5}=($ liquidity on hand) $:$ (cash and deposits on hand) $/$ (sales)

$z_{6}:$ (sales)/(fixed assets)

Evaluation results are shown in Tables 6-11.

Table 6: The second Comparison among efficiency measures for efficient DMUs in data group (1)

\begin{tabular}{|c|c|c|c|}
\hline & $\alpha_{\max }$ & Super-efficiency & Proposed measure \\
\hline Mabuchi-Motor & $96.82 \%$ & 2.43 & 1.59 \\
\hline Teak & $66.73 \%$ & 1.75 & 1.48 \\
\hline msc.panasonic & $0.47 \%$ & 1.25 & 1.24 \\
\hline Sony & $1.10 \%$ & 1.24 & 1.23 \\
\hline mke.panasonic & $0.00 \%$ & 1.06 & 1.06 \\
\hline
\end{tabular}

Table 7: The second comparison among efficiency measures for efficient DMUs in data group (2)

\begin{tabular}{|c|c|c|c|}
\hline & $\alpha_{\max }$ & Super-efficiency & Proposed measure \\
\hline Hankyu-Dept & $51.85 \%$ & 1.923 & 1.932 \\
\hline Hanshin & $33.05 \%$ & 1.587 & 1.445 \\
\hline Isetan & $2.94 \%$ & 1.359 & 1.33 \\
\hline Meitetsu-Dept & $0.45 \%$ & 1.241 & 1.258 \\
\hline Maruei & $0.01 \%$ & 1.156 & 1.157 \\
\hline Daiwa & $0.01 \%$ & 1.114 & 1.128 \\
\hline Nagano-Tokyu & $0.01 \%$ & 1.132 & 1.121 \\
\hline Iwataya & $0.00 \%$ & 1.108 & 1.106 \\
\hline Matsuya & $0.00 \%$ & 1.064 & 1.078 \\
\hline
\end{tabular}

Table 8: The second comparison among efficiency measures for efficient DMUs in data group (3)

\begin{tabular}{|c|c|c|c|}
\hline & $\alpha_{\max }$ & Super-efficiency & Proposed measure \\
\hline Itoyokado & $7.41 \%$ & 1.482 & 1.396 \\
\hline Taiyo & $0.00 \%$ & 1.133 & 1.131 \\
\hline Aoki Super & $0.00 \%$ & 1.104 & 1.094 \\
\hline Yorkbenimaru & $0.00 \%$ & 1.079 & 1.073 \\
\hline MatsumotoKiyoshi & $0.00 \%$ & 1.06 & 1.06 \\
\hline Harashin & $0.00 \%$ & 1.008 & 1.008 \\
\hline
\end{tabular}

In these tables Sogo and Mycal which went down are evaluated very lowly. This means that selection of indices in this section is better than Sec. 5.1, but we cannot recommend this selection easily, because [9] in year 2003 uses different indices from [8] in year 1993 as Z-values (See [1]) which predict corporate bankruptcy. 
Table 9: Efficiency scoress for inefficient DMUs in data group

(1)

\begin{tabular}{|c|c|c|c|}
\hline Marantz & 0.98 & Yokowo & 0.60 \\
\hline kme.panasonic & 0.93 & Clarion & 0.56 \\
\hline Foster & 0.87 & Matsushita & 0.55 \\
\hline Aiwa & 0.85 & Sharp & 0.53 \\
\hline JvcVictor & 0.78 & Melco & 0.53 \\
\hline Enplas & 0.77 & NEC & 0.52 \\
\hline Toshibatec & 0.77 & Sanyo & 0.52 \\
\hline TOA & 0.77 & Oki & 0.51 \\
\hline mrc.panasonic & 0.74 & Toshiba & 0.50 \\
\hline Rion & 0.66 & Fujielectric & 0.50 \\
\hline Columbia & 0.65 & Hitachi & 0.48 \\
\hline Zojirushi & 0.62 & Fujitsu & 0.47 \\
\hline Pioneer & 0.62 & Nakamichi & 0.18 \\
\hline
\end{tabular}

Table 10: Efficiency scoress for inefficient DMUs in data group (2)

\begin{tabular}{|c|c|c|c|}
\hline Tokyu-Dept & 0.985 & Platz-Kintetsu & 0.744 \\
\hline Daimaru & 0.899 & Saikaya & 0.687 \\
\hline Matsuzakaya & 0.820 & Sanyo-Dept & 0.677 \\
\hline Takashimaya & 0.768 & Izutsuya & 0.554 \\
\hline Mitsukoshi & 0.768 & Sogo & 0.257 \\
\hline
\end{tabular}

Table 11: Efficiency scoress for inefficient DMUs in data group (3)

\begin{tabular}{|c|c|c|c|}
\hline Wellmart & 0.988 & Fuji & 0.566 \\
\hline Marukyo & 0.922 & Daiei & 0.565 \\
\hline HacKimisawa & 0.895 & DOMY & 0.551 \\
\hline Maruya & 0.856 & TobuStore & 0.547 \\
\hline Inageya & 0.809 & Seiyu & 0.533 \\
\hline heiwado & 0.788 & Uny & 0.524 \\
\hline Yamazawa & 0.767 & Okuwa & 0.503 \\
\hline Lifecorp & 0.743 & Jusco & 0.501 \\
\hline SuperDaiei & 0.700 & Marukyu & 0.477 \\
\hline U-Store & 0.689 & Maruetsu & 0.472 \\
\hline Maruwa & 0.670 & Izumi & 0.462 \\
\hline Jujiya & 0.668 & Flex & 0.442 \\
\hline Tokyu Store & 0.658 & Kotobukiya & 0.435 \\
\hline Yamanaka & 0.624 & Izumiya & 0.428 \\
\hline Valor & 0.590 & Mycal & 0.424 \\
\hline Kansai Super & 0.588 & Tenmaya-Store & 0.382 \\
\hline
\end{tabular}




\section{Discussion}

Each method used in this paper is well known, but their combinational usage is original.

We propose use of the Kalman filter for estimation of variances which is needed in the stochastic efficiency model but is difficult on a single time point evaluation. When there are longitudinal data, estimation of variance is naturally included in the Kalman filter, for example, in Equation (10). Of course we can apply formulations different from Equation (1) to Equation (7).

In a case of single input, efficient scores in DEA are given by a linear function of outputs/input and the function can be used as a discriminatory function if a cut-off point is determined. However, DEA is a method which stresses merits of each DMU and takes weakness of them lightly. Thus, there may be no problem of evaluation of DMUs with good performance, but for DMUs with worse performance we may be unable to predict their insolvency. In fact Sogo and Mycal went down, but they were evaluated as efficient DMUs in Sec.5.1. We also have a problem which indices should be selected because Sec.5.2. obtained a different efficiency score from Sec.5.1. for the same firm.

We think various methods should be used for evaluation of firms, according to purposes of analysis.

\section{References}

[1] E.I. Altman: Financial ratios, discriminant analysis and the prediction of corporate bankruptcy. The Journal of Finance, 23 (1968), 589-609.

[2] P. Andersen and N.C. Petersen: A procedure for ranking efficient units in Data Envelopment Analysis. Management Science, 39 (1993), 1261-1264.

[3] P.J. Brockwell and R.A. Davis: Introduction to Time Series and Forecasting, Second ed. (Springer-Verlag, 2002), Chap.8.

[4] A. Charnes, W.W. Cooper, and E. Rhodes: Measuring efficiency of decision making units. European Journal of Operational Research, 2 (1978), 429-444.

[5] G. Kitagawa and W. Gersch: A smoothness priors-state space modeling of time series with trend and seasonality. Journal of the American Statistical Association, 79 (1984), 378-389.

[6] A. Koyama: Finance and Decision Making (Asakura, 2001) (in Japanese).

[7] H. Morita and L.M. Seiford: Characteristics on stochastic DEA efficiency -reliability and probability being efficient-. Journal of the Operations Research Society of Japan, 42 (1999), 389-404.

[8] Toyo Keizai Inc.: The Weekly Toyo keizai, 5175 (23, Aug., 1993) (in Japanese).

[9] Toyo Keizai Inc.: The Weekly Toyo keizai, (27, Sept., 2003) (in Japanese).

Tohru Ueda

Department of Computer and Information Science

Faculty of Science and Technology

Seikei University

3-3-1, Kichijoji-Kitamachi, Musasino-shi

Tokyo, 180-8633 Japan

E-mail: ueda@st.seikei.ac.jp 